\title{
REMARKS ON GRASSMANNIAN SUPERMANIFOLDS
}

\author{
OSCAR ADOLFO SÁNCHEZ VALENZUELA
}

\begin{abstract}
This paper studies some aspects of a particular class of examples of supermanifolds; the supergrassmannians, introduced in [Manin]. Their definition, in terms of local data and glueing isomorphisms, is reviewed. Explicit formulas in local coordinates are given for the Lie group action they come equipped with. It is proved that, for those supergrassmannians whose underlying manifold is an ordinary grassmannian, their structural sheaf can be realized as the sheaf of sections of the exterior algebra bundle of some canonical vector bundle. This realization holds true equivariantly for the Lie group action in question, thus making natural in these cases the identification given in [Batchelor]. The proof depends on the computation of the transition functions of the supercotangent bundle as defined in a previous work [OASV 2]. Finally, it is shown that there is a natural supergroup action involved (in the sense of [OASV 3]) and hence, the supergrassmannians may be regarded as examples of superhomogeneous spaces-a notion first introduced in [Kostant]. The corresponding Lie superalgebra action can be realized as superderivations of the structural sheaf; explicit formulas are included for those supergrassmannians identifiable with exterior algebra vector bundles.
\end{abstract}

Introduction. There are various definitions of supermanifolds in use. Mathematicians define a $C^{\infty}$ (resp., holomorphic) supermanifold as a pair, $\left(M, \mathscr{A}_{M}\right)$, consisting of an ordinary $C^{\infty}$ (resp., holomorphic) manifold $M$, together with a sheaf $\mathscr{A}_{M}$ of supercommutative superalgebras over $\mathbf{R}$ (resp., C) satisfying certain conditions. The various definitions in the literature correspond to the various conditions imposed on the structural sheaf. There seem to be at least two streams of approach, which one may vaguely refer to as algebraic and differential, respectively (see, for example, [Kostant], [Leĭtes] and [Manin] for the first and [Rogers], [Boyer and Gitler] and [Jadczyk and Pilch] for the second; for a unified view and a generalization that uses a nontrivial underlying super ring, see [Rothstein 2]).

CONVENTION. For the purposes of this paper, we shall be concrete and shall understand the definition of supermanifold as in [Manin].

As far as the first approach is concerned, it is a theorem of Batchelor [Batchelor] that, in the $C^{\infty}$ case, the sheaf $\mathscr{A}_{M}$ can be realized as the sheaf of sections, $\Gamma(\cdot, \bigwedge E)$, of the exterior algebra bundle, $\bigwedge E$, of some vector bundle $E \rightarrow M$ over $M$. This identification is not categorical, however: it depends on choices; besides, supermanifold morphisms are more general than vector bundle maps, a fact pointed out in [Leĭtes], and it is known that in the holomorphic case there are obstructions to such an identification (see, for example, [Manin] and [Rothstein 1]).

Received by the editors March $18,1987$.

1980 Mathematics Subject Classification (1985 Revision). Primary 58A50.

This work is part of the Ph.D. thesis submitted by the author to the Department of Mathematics of Harvard University (May 1986). 
In the physics literature, on the other hand, one frequently finds supermanifolds defined by the specification that the odd variables are spinors. What this means, presumably, is the following: Let $G$ be some group acting on the manifold $M$ and as bundle automorphisms of a vector bundle $E \rightarrow M$. Then $G$ has an induced action on $\Gamma(\cdot, \bigwedge E)$ and hence acts as a group of automorphisms of the corresponding supermanifold. If $M$ is a spin manifold and $E \rightarrow M$ is the spin bundle, this then specifies the supermanifold structure as a $G$-space.

The case of the conformally compactified and complexified Minkowski space, $M$, provides us with an important example (see [Guillemin and Sternberg] for details): $M$ itself can be regarded as the space $G_{2}\left(\mathbf{C}^{4}\right)$ of two-dimensional (complex) subspaces of $\mathbf{C}^{4}$. The spin bundle then becomes identified with the tautological bundle $E \rightarrow G_{2}\left(\mathbf{C}^{4}\right)$ whose fiber at $u \in G_{2}\left(\mathbf{C}^{4}\right)$ is just the two-dimensional space $E_{u}$ represented by $u$. The group $G=\mathrm{GL}(4, \mathbf{C})$ acts as bundle automorphisms, and the picture is now the one of the preceding paragraph. This obviously generalizes to $M=G_{k}\left(V^{m}\right)$, the Grassmannian manifold of $k$-planes of some $m$-dimensional vector space $V^{m}$, and $G=\mathrm{GL}(m)$ acting as bundle automorphisms of the rank $k$ tautological bundle $E \rightarrow G_{k}\left(V^{m}\right)$.

In the supermanifold setting we may consider the class of supergrassmannians introduced in [Manin]: the supergrassmannian $\mathbf{G}_{k \mid h}\left(V^{m \mid n}\right)$ arises as the supermanifold associated to the set of $(k, h)$-dimensional supervector subspaces, $u$, of a given $(m, n)$-dimensional supervector space $V^{m \mid n}\left(=V_{0}^{m} \oplus V_{1}^{n}\right)$; that is,

$$
u \subset V^{m \mid n} ; \quad \operatorname{dim}\left(u \cap V_{0}^{m}\right)=k \quad \text { and } \quad \operatorname{dim}\left(u \cap V_{1}^{n}\right)=h .
$$

It turns out that the underlying manifold of $\mathbf{G}_{k \mid h}\left(V^{m \mid n}\right)$ is just $G_{k}\left(V_{0}^{m}\right) \times G_{h}\left(V_{1}^{n}\right)$, and its odd dimension is $h(m-k)+k(n-h)$. Furthermore, since the Lie group $\mathrm{GL}\left(V_{0}^{m}\right) \times \mathrm{GL}\left(V_{1}^{n}\right)$ clearly operates or the set of $(k, h)$-dimensional supervector subspaces of $V^{m \mid n}$, one obtains a group homomorphism

$$
\mathrm{GL}\left(V_{0}^{m}\right) \times \mathrm{GL}\left(V_{1}^{n}\right) \rightarrow \operatorname{Aut} \mathscr{A}_{\mathbf{G}_{k \mid h}\left(V^{m \mid n}\right)} .
$$

On suitable open superdomains $\mathscr{U} \subset \mathbf{G}_{k \mid h}\left(V^{m \mid n}\right)$, this action is nothing but the generalization of

$$
z \mapsto(A z+B)(C z+D)^{-1} ; \quad\left(\begin{array}{cc}
A & B \\
C & D
\end{array}\right) \in \mathrm{GL}\left(V_{0}^{m}\right)
$$

i.e., the action of $\mathrm{GL}\left(V_{0}^{m}\right)$ on (suitable open sets of) the ordinary grassmannian $G_{k}\left(V_{0}^{m}\right)$.

Just as in ordinary differential geometry, the supergrassmannians come equipped with two canonical supervector bundles, constructed via transition functions as in [OASV 2]: $\mathbf{E}_{k \mid h}=\left(E, \mathscr{A}_{E}\right)$ of type $(k \mid h)$ and $\mathbf{F}_{m-k \mid n-h}=\left(F, \mathscr{A}_{F}\right)$ of type $(m-k \mid n-h)$. As a pleasant result, there is a canonical identification between the supercotangent bundle $\mathbf{S} \mathbf{T}^{*} \mathbf{G}_{k \mid h}\left(V^{m \mid n}\right)$ and the supermanifold of all the supervector bundle morphisms $\mathbf{F}_{m-k \mid n-h} \rightarrow \mathbf{E}_{k \mid h}$; i.e.,

$$
\mathbf{S T}^{*} \mathbf{G}_{k \mid h}\left(V^{m \mid n}\right) \simeq \mathscr{H} a m\left(\mathbf{F}_{m-k \mid n-h}, \mathbf{E}_{k \mid h}\right) .
$$

In particular, the transition functions of this supervector bundle can be used to prove that, with respect to the $\mathrm{GL}\left(V_{0}^{m}\right) \times \mathrm{GL}\left(V_{1}^{n}\right)$-action on $\mathrm{G}_{k \mid h}\left(V^{m \mid n}\right)$ above and within the spirit of the theorem of Batchelor, the following is true. 
THEOREM. (a) $\mathbf{G}_{k \mid 0}\left(V^{m \mid n}\right)=\left(G_{k}\left(V_{0}^{m}\right), \mathscr{A}_{G_{k}\left(V_{0}^{m}\right)}\right)$ is a supermanifold of dimension $(k(m-k), n k)$ for which an equivariant identification

$$
\mathscr{A}_{G_{k}\left(V_{0}^{m}\right)} \stackrel{\sim}{\longrightarrow} \mathscr{G}_{r}\left(\mathscr{A}_{G_{k}\left(V_{0}^{m}\right)}\right) \simeq \Gamma\left(\cdot, \bigwedge\left(V_{1} \otimes E_{1}\right)\right)
$$

exists. (Here $E_{0} \rightarrow G_{k}\left(V_{0}^{m}\right)$ denotes the rank- $k$ tautological vector bundle over the grassmannian $G_{k}\left(V_{0}^{m}\right) ; V_{1}^{n} \rightarrow G_{k}\left(V_{0}^{m}\right)$ is the rank-n trivial bundle, and $V_{1}^{n} \otimes E_{0} \rightarrow$ $G_{k}\left(V_{0}^{m}\right)$ is the tensor product bundle.)

REMARK. A similar result holds for the $(k(m-k), n k)$-dimensional supermanifold $\mathbf{G}_{0 \mid k}\left(V^{n \mid m}\right)=\left(G_{k}\left(V_{1}^{m}\right), \mathscr{A}_{G_{k}\left(V_{1}^{m}\right)}\right)$, in which case we have $\mathscr{A}_{G_{k}\left(V_{1}^{m}\right)} \stackrel{\sim}{\longrightarrow}$ $\mathscr{G}_{r}\left(\mathscr{A}_{G_{k}\left(V_{1}^{m}\right)}\right) \simeq \Gamma\left(\cdot, \bigwedge\left(V_{0} \otimes E_{1}\right)\right)$, equivariantly.

(b) $\mathbf{G}_{k \mid n}\left(V^{m \mid n}\right)=\left(G_{k}\left(V_{0}^{m}\right), \mathscr{A}_{G_{k}\left(V_{0}^{m}\right)}\right)$ is a supermanifold of dimension $(k(m-k), n(m-k))$ for which an equivariant identification

$$
\mathscr{A}_{G_{k}\left(V_{0}^{m}\right)} \stackrel{\sim}{\longrightarrow} \mathscr{G}_{r}\left(\mathscr{A}_{G_{k}\left(V_{0}^{m}\right)}\right) \simeq \Gamma\left(\cdot, \bigwedge\left(V_{1} \otimes F_{0}^{*}\right)\right)
$$

exists. (Here $F_{0}^{*} \rightarrow G_{k}\left(V_{0}^{m}\right)$ denotes the rank- $(m-k)$ tautological vector bundle over the grassmannian $G_{k}\left(V_{0}^{m}\right)$.)

REMARK. Similarly, for $\mathbf{G}_{n \mid k}\left(V^{n \mid m}\right)=\left(G_{k}\left(V_{1}^{m}\right), \mathscr{A}_{G_{k}\left(V_{1}^{m}\right)}\right)$, we have $\mathscr{A}_{G_{k}\left(V_{1}^{m}\right)}$ $\stackrel{\sim}{\longrightarrow} \mathscr{G}_{\mu}\left(\mathscr{A}_{G_{k}\left(V_{1}^{m}\right)}\right) \simeq \Gamma\left(\cdot, \bigwedge\left(V_{0} \otimes F_{1}^{*}\right)\right)$, equivariantly.

In particular, these results already cover the following special cases:

(i) All the supergrassmannians whose odd dimension is 1 .

(ii) All the superprojective spaces (i.e., those supergrassmannians whose underlying manifold is a projective space), regardless of their odd dimension.

However, as soon as one considers supergrassmannians $\mathbf{G}_{k \mid h}\left(V^{m \mid n}\right)$ with nontrivial underlying factors (i.e., for which neither $G_{k}\left(V_{0}^{m}\right)$, nor $G_{h}\left(V_{1}^{n}\right)$ reduce to a single point) there is no way of identifying $\mathscr{A}_{\mathbf{G}_{k \mid h}\left(V^{m \mid n}\right)}$ with $\mathscr{G}_{r} \mathscr{A}_{\mathbf{G}_{k \mid h}\left(V^{m \mid n}\right)}$ in a $\mathrm{GL}\left(V_{0}^{m}\right) \times \mathrm{GL}\left(V_{1}^{n}\right)$-equivariant manner. An explicit counterexample is provided by the supergrassmannian $\mathrm{G}_{1 \mid 1}\left(V^{2 \mid 2}\right)$. This can be proved by means of a pedestrian use of the formulas developed in this paper. We remark, however, that there is a more satisfactory approach to this point due to $M$. Rothstein via the computation of certain Lie algebra cohomology classes [Rothstein 3]. At any rate, this result shows that under a Lie group action, Batchelor's Theorem does not reduce the theory of supermanifolds to that of vector bundles, even in the $C^{\infty}$ category.

Finally, let us note that (2) is just an ordinary Lie group action. It would be desirable, however, to prove that the supergrassmannians are very natural examples of superhomogeneous supermanifolds (a notion already defined in [Kostant]). Based on some of our considerations in [OASV 3] on the one hand, and on the work of Kostant on the other, we may prove that the infinitesimal version of this action is what it should be; more precisely,

PROPOSITION. There is a graded Lie algebra homomorphism $\mathfrak{g l}\left(V_{0}^{m} \mid V_{1}^{n}\right) \rightarrow$ Der $\mathscr{A}_{\mathbf{G}_{\boldsymbol{k} \mid \boldsymbol{h}}\left(V^{m \mid n}\right)}$ whose restriction to $\mathfrak{g l}\left(V_{0}^{m}\right) \oplus \mathfrak{g l}\left(V_{1}^{n}\right)=\left(\mathfrak{g l}\left(V_{0}^{m} \mid V_{1}^{n}\right)\right)_{0}$ coincides with the (classical) infinitesimal action obtained from (2) above.

The Lie supergroup that acts on the supergrassmannian $\mathbf{G}_{k \mid h}\left(V^{m \mid n}\right)$ is

$$
\left\{S\left(\text { End } V^{m \mid n}\right)\right\}^{*},
$$


the subsupermanifold of $S\left(\right.$ End $\left.V^{m \mid n}\right)$ described in local coordinates by the condition of having a nonzero Berezinian. The underlying Lie group is just $\operatorname{GL}\left(V_{0}^{m}\right) \times$ $\mathrm{GL}\left(V_{1}^{n}\right)$ and its odd dimension is $2 \operatorname{dim} V_{0}^{m} \operatorname{dim} V_{1}^{n}$, as pointed out in [OASV 3]. The computations in this work suggest there is indeed a notion of maximal parabolic subsupergroup, $\mathscr{P}_{k \mid h}\left(V^{m \mid n}\right)$, satisfying the correct dimensionality relations; namely,

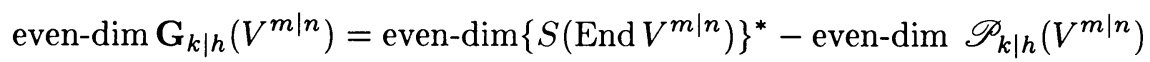
and

odd-dim $\mathbf{G}_{k \mid h}\left(V^{m \mid n}\right)=$ odd-dim $\left\{S\left(\text { End } V^{m \mid n}\right)\right\}^{*}$ - odd-dim $\mathscr{P}_{k \mid h}\left(V^{m \mid n}\right)$.

ACKNOWLEDGMENTS. This work was made possible by the advice and guidance of my teacher, Shlomo Sternberg, to whom I am deeply grateful; he has been a source of continual inspiration. The results here constituted a part of the Ph.D. thesis I submitted to the Department of Mathematics of Harvard University. I would also like to thank V. Guillemin and J. Wolf for their reading of the original version of this work and $B$. Kostant for his influence through his lectures.

1. Supergrassmannians. Let $V^{m \mid n}=V_{0}^{m} \oplus V_{1}^{n}$ be a given $(m, n)$-dimensional supervector space over $\mathbf{R}$ (or $\mathbf{C}$ ), to be kept fixed throughout this section. Let us assume that we are given some $(m-k, n-h)$-dimensional supervector subspace, say $W=W_{0} \oplus W_{1}$, of $V^{m \mid n}$. This means that $W_{\mu}=W \cap V_{\mu} \subset V_{\mu}(\mu=0,1)$ and the ordinary vector space exact sequence

$$
0 \rightarrow W \rightarrow V^{m \mid n} \rightarrow V^{m \mid n} / W \rightarrow 0
$$

takes the form

$$
0 \rightarrow W_{0} \oplus W_{1} \underset{j}{\rightarrow} V_{0}^{m} \oplus V_{1}^{n} \underset{\pi}{\longrightarrow}\left(V_{0}^{m} / W_{0}\right) \oplus\left(V_{1}^{n} / W_{1}\right) \rightarrow 0
$$

where both the injection $j$ and the projection $\pi$ are even homomorphisms (cf. [Corwin et al.] and [OASV 1]).

It is well known that if $\hat{\mathscr{U}}_{W}$ denotes the set of all vector subspaces of $V^{m \mid n}$ complementary to $W$ and a choice $\hat{u} \in \hat{\mathscr{U}}_{W}$ is made, a bijection

$$
\hat{\mathscr{U}}_{W} \rightarrow \operatorname{Hom}\left(V^{m \mid n} / W, W\right)
$$

exists under which $\hat{u}$ corresponds to the zero map. Even though we can always choose $\hat{u}$ to be a $(k, h)$-dimensional supervector subspace of $V^{m \mid n}$, it is clear that not every element of $\hat{\mathscr{U}}_{W}$ has a direct sum decomposition $u_{0} \oplus u_{1}$ with $u_{0} \subset V_{0}^{m}$ and $u_{1} \subset V_{1}^{n}$. However, we can detect precisely what the complementary supervector subspaces to $W$ are, by just looking at the supervector space structure of $\operatorname{Hom}\left(V^{m \mid n} / W, W\right)$. More precisely, we have the following rather obvious fact:

1.1 PROPOSITION. If $\hat{u}$ is chosen to be a $(k, h)$-dimensional supervector subspace of $V^{m \mid n}$, then (1.3) yields a one-to-one correspondence between the set of all $(k, h)$-dimensional supervector subspaces of $V^{m \mid n}$ and the even subspace $\left(\operatorname{Hom}\left(V^{m \mid n} / W, W\right)\right)_{0}$.

Now, according to the theory developed in [Kostant] and [Leites], one can associate to the supervector space

$$
\operatorname{Hom}\left(V^{m \mid n} / W, W\right)=\left(\operatorname{Hom}\left(V^{m \mid n} / W, W\right)\right)_{0} \oplus\left(\operatorname{Hom}\left(V^{m \mid n} / W, W\right)\right)_{1}
$$


a (super-) affine supermanifold, $\left(\left(\operatorname{Hom}\left(V^{m \mid n} / W, W\right)\right)_{0}, \mathscr{A}\right)$, by letting the sheaf $\mathscr{A}$ be given by

$$
\mathscr{A}=\left.\mathscr{C}^{\infty}\right|_{\left(\operatorname{Hom}\left(V^{m \mid n} / W, W\right)\right)_{0}} \otimes \bigwedge\left(\left[\left(\operatorname{Hom}\left(V^{m \mid n} / W, W\right)\right)_{1}\right]^{*}\right)
$$

Note that

$$
\begin{aligned}
\left(\operatorname{Hom}\left(V^{m \mid n} / W, W\right)\right)_{0} & \simeq \operatorname{Hom}\left(V_{0}^{m} / W_{0}, W_{0}\right) \oplus \operatorname{Hom}\left(V_{1}^{n} / W_{1}, W_{1}\right) \\
& \simeq \mathscr{U}_{W_{0}} \times \mathscr{U}_{W_{1}}
\end{aligned}
$$

where $\mathscr{U}_{W_{0}}$ (resp., $\mathscr{U}_{W_{1}}$ ) is the open subset of $G_{k}\left(V_{0}^{m}\right)$ (resp., $G_{h}\left(V_{1}^{n}\right)$ ) consisting of those $k$ (resp., $h$ ) dimensional subspaces complementary to $W_{0}$ (resp., $W_{1}$ ). That is, $\mathscr{U}_{W_{0}} \times \mathscr{U}_{W_{1}} \subset G_{k}\left(V_{0}^{m}\right) \times G_{h}\left(V_{1}^{n}\right)$ and this observation suggests to try to cover $G_{k}\left(V_{0}^{m}\right) \times G_{h}\left(V_{1}^{n}\right)$ by open sets of the form $\mathscr{U}_{I_{0}} \times \mathscr{U}_{I_{1}}$ over each of which we will have a sheaf $\mathscr{A}_{\left(I_{0}, I_{1}\right)}$ of supercommutative superalgebras given by (1.5) and then provide appropriate glueing isomorphisms $\varphi_{\left(I_{0}, I_{1}\right)\left(J_{0}, J_{1}\right)}$ so as to end up with a supermanifold $\left(G_{k}\left(V_{0}^{m}\right) \times G_{h}\left(V_{1}^{n}\right), \mathscr{A}\right)$ whose underlying $C^{\infty}$ (resp., holomorphic) manifold is precisely the space of $(k, h)$-dimensional supervector subspaces of $V^{m \mid n}$, namely, the product $G_{k}\left(V_{0}^{m}\right) \times G_{h}\left(V_{1}^{n}\right)$.

We are thus led to Manin's prescription [Manin]: The indexing set for the basic superdomains $\mathscr{U}_{I}$ will now consist of pairs of disjoint ordered sequences

$$
I=I_{0} \hat{\cup} I_{1} \quad(\hat{\cup}:=\text { disjoint union })
$$

of length $m-k$ and $n-h$, respectively. (Note. The interpretation of such an indexing can be given in terms of some definite choice of homogeneous (ordered) basis $\left\{\mathbf{e}_{i}\right\}$ of $V_{0}^{m} \oplus V_{1}^{n}$ by letting $W_{I}$ be the $(m-k, n-h)$-dimensional supervector subspace $\operatorname{Span}\left\{\mathbf{e}_{i}: i \in I=I_{0} \hat{\cup} I_{1}\right\}$.) Then we put

$$
\mathscr{U}_{I}:=\mathscr{U}_{I_{0}} \times \mathscr{U}_{I_{1}}
$$

and let

$$
\mathscr{A}_{I}:=\mathscr{C}^{\infty} \mid \mathscr{U}_{I_{0}} \times \mathscr{U}_{I_{1}} \otimes \bigwedge\left(\left(\operatorname{Hom}\left(V_{0}^{m} / W_{I_{0}}, W_{I_{1}}\right) \oplus \operatorname{Hom}\left(V_{1}^{n} / W_{I_{1}}, W_{I_{0}}\right)\right)^{*}\right)
$$

Following [Manin] we shall arrange the coordinate functions of the superdomain $\left(\mathscr{U}_{I}, \mathscr{A}_{I}\right)$ into a $[(m-k)+(n-h)] \times[m+n]$ matrix, $p_{I}=\left(p_{I}^{i a}\right)$, where, if $I^{c}=I_{0}^{c} \hat{\cup} I_{1}^{c}$ denotes the complementary ordered sequence of length $(k, h)$,

$$
p_{I}^{i a}= \begin{cases}\delta^{i a} ; & i \in I, a \in I, \\ z_{I}^{i a} ; & i \in I, a \in I^{c},\end{cases}
$$

and additionally,

$$
z_{I}^{i a}= \begin{cases}x_{I}^{i a} ; & i \in I_{0}, a \in I_{0}^{c}, \\ y_{I}^{i a} ; & i \in I_{1}, a \in I_{1}^{c}, \\ \varsigma_{I}^{i a} ; & i \in I_{1}, a \in I_{0}^{c} \\ \xi_{I}^{i a} ; & i \in I_{0}, a \in I_{1}^{c}\end{cases}
$$

where the $x_{I}^{i a}$ (resp., $y_{I}^{i a}$ ) are the standard coordinate functions on $\mathscr{U}_{I_{0}}$ (resp., $\mathscr{U}_{I_{1}}$ ) and the $\varsigma_{I}^{i a}$ (resp., $\left.\xi_{I}^{i a}\right)$ are the generators of $\Lambda\left(\left(\operatorname{Hom}\left(V_{0}^{m} / W_{I_{0}}, W_{I_{1}}\right)\right)^{*}\right)$ [resp., $\left.\bigwedge\left(\left(\operatorname{Hom}\left(V_{1}^{n} / W_{I_{1}}, W_{I_{0}}\right)\right)^{*}\right)\right]$. We shall also write the matrix $\left(p_{I}^{i a}\right)$ in the symbolic form

$$
\begin{aligned}
& p_{I}=\left(\begin{array}{cccc}
\mathrm{id} & x_{I} & 0 & \xi_{I} \\
0 & \zeta_{I} & \mathrm{id} & y_{I}
\end{array}\right) \begin{array}{l}
\} I_{0} \\
I_{1}
\end{array} \\
& \begin{array}{llll}
I_{0} & I_{0}^{c} & I_{1} & I_{1}^{c}
\end{array}
\end{aligned}
$$


If $J=J_{0} \hat{\cup} J_{1}$ is some other sequence, we define $g_{I J}$ as the submatrix of $p_{I}$ obtained after deleting the columns indexed by $J^{c}=J_{0}^{c} \hat{\cup} J_{1}^{c}$. This yields an $(m-k)+(n-h)$ square even matrix

$$
\left.g_{I J}=\left(\begin{array}{cc}
g_{I_{0} J_{0}} & g_{I_{0} J_{1}} \\
g_{I_{1} J_{0}} & g_{I_{1} J_{1}}
\end{array}\right)\right\} \begin{gathered}
I_{0} \\
I_{1} \\
J_{0} \\
J_{1}
\end{gathered}
$$

As we know from [Kostant] and [Lertes], this matrix is invertible if and only if $g_{I_{0} J_{0}}$ and $g_{I_{1} J_{1}}$ are, and this fact guarantees that the glueing of sheaves below takes place precisely over $\mathscr{U}_{I_{0}} \cap \mathscr{U}_{J_{0}} \times \mathscr{U}_{I_{1}} \cap \mathscr{U}_{J_{1}}=\mathscr{U}_{I} \cap \mathscr{U}_{J}$. So define

$$
\varphi_{I J}: \mathscr{A}_{I}\left|\mathscr{U}_{I} \cap \mathscr{U}_{J} \stackrel{\sim}{\longrightarrow} \mathscr{A}_{J}\right| \mathscr{U}_{I} \cap \mathscr{U}_{J}
$$

by letting

$$
\varphi_{I J} z_{I}^{i a}=\left(\left(g_{J I}\right)^{-1} \cdot p_{J}\right)^{i a}
$$

It is not difficult to check that the $\varphi_{I J}$ 's are sheaf isomorphisms with $\left(\varphi_{I J}\right)^{-1}=$ $\varphi_{J I}, \varphi_{I I}=\mathrm{id}$ and $\varphi_{J K} \varphi_{I J}=\varphi_{I K}$ on $\mathscr{U}_{I} \cap \mathscr{U}_{J} \cap \mathscr{U}_{K}$, so that there exists a unique sheaf $\mathscr{A}$ of supercommutative superalgebras on $G_{k}\left(V_{0}^{m}\right) \times G_{h}\left(V_{1}^{n}\right)$, such that

$$
\mathrm{G}_{k \mid h}\left(V^{m \mid n}\right)=\left(G_{k}\left(V_{0}^{m}\right) \times G_{h}\left(V_{1}^{n}\right), \mathscr{A}\right)
$$

becomes a $(k(m-k)+h(n-h), h(m-k)+k(n-h))$-dimensional $C^{\infty}$ (resp., holomorphic) supermanifold. Furthermore, it is clear from this construction that

$$
\mathscr{G}_{r}^{0} \mathscr{A}=\mathscr{A} / \mathscr{J} \cong \mathscr{C}^{\infty} \mid G_{k}\left(V_{0}^{m}\right) \times G_{h}\left(V_{1}^{n}\right)
$$

where $\mathcal{J}$ denotes the ideal generated by the odd elements. On the other hand, it has been proved in [Manin] that

$$
\mathscr{G}_{r} \mathscr{A} \simeq \Gamma\left(\cdot, \bigwedge\left(\left[\left(F_{1}\right)^{*} \otimes E_{0}\right] \oplus\left[\left(F_{0}\right)^{*} \otimes E_{1}\right]\right)\right)
$$

where $E_{0}$ (resp., $E_{1}$ ) denotes the total space of the rank $k$ (resp., $h$ ) tautological bundle over $G_{k}\left(V_{0}^{m}\right)$ (resp., $G_{h}\left(V_{1}^{n}\right)$ ), while $\left(F_{0}\right)^{*}$ (resp., $\left.\left(F_{1}\right)^{*}\right)$ denotes that of the dual of the rank $m-k$ (resp., $n-h$ ) tautological bundle over $G_{k}\left(V_{0}^{m}\right)$ (resp., $G_{h}\left(V_{1}^{n}\right)$ ). The proof consists of using the local data in terms of which the supergrassmannians have been defined in conjunction with the identifications

$$
\begin{aligned}
& \left(\operatorname{Hom}\left(V_{0}^{m} / W_{I_{0}}, W_{I_{1}}\right)\right)^{*} \simeq\left(W_{I_{1}}\right)^{*} \otimes\left(V_{0}^{m} / W_{I_{0}}\right), \\
& \left(\operatorname{Hom}\left(V_{1}^{n} / W_{I_{1}}, W_{I_{0}}\right)\right)^{*} \simeq\left(W_{I_{0}}\right)^{*} \otimes\left(V_{1}^{n} / W_{I_{1}}\right),
\end{aligned}
$$

although the details will be omitted here (see $\S 3$, however).

2. Homogeneous space structure. From the geometric interpretation given in $\S 1$, it is clear that the group $\mathrm{GL}(m) \times \mathrm{GL}(n)$, acting on $V^{m \mid n}$ in the usual way, will transform $(k, h)$-dimensional supervector subspaces into $(k, h)$-dimensional supervector subspaces, so that it is only natural to try to obtain a representation

$$
\rho: \mathrm{GL}(m) \times \mathrm{GL}(n) \rightarrow \operatorname{Aut} \mathscr{A}
$$

for which

$$
\operatorname{Gr}(\rho): \operatorname{GL}(m) \times \mathrm{GL}(n) \rightarrow \operatorname{Aut}\left(\mathscr{G}_{\mu} \mathscr{A}\right)
$$


reduces to the usual action. The prescription for this effect goes as follows (cf. [Manin]): given $g \in \mathrm{GL}(m) \times \mathrm{GL}(n)$ we consider $p_{I} g^{-1}$ and look for the invertible submatrix $\psi_{I \tilde{I}}$ obtained from the columns of $p_{I} g^{-1}$ indexed by $\tilde{I}=\tilde{I}_{0} \hat{\cup} \tilde{I}_{1}$ (one may regard this as the definition of $\tilde{I}$ ). Then we set

$$
\rho_{I \tilde{I}}(g): \mathscr{A}\left|\mathscr{V}_{I} \rightarrow \mathscr{A}\right| \mathscr{V}_{\tilde{I}}
$$

where $\mathscr{V}_{I}$ and $\mathscr{V}_{\tilde{I}}$ are the open subsets of $\mathscr{U}_{I}$ and $\mathscr{U}_{\tilde{I}}$ determined by the condition of $\psi_{I \tilde{I}}$ being invertible (see below). Explicitly, if we write $p_{I}$ as in (11) and for $g \in \mathrm{GL}(m) \times \mathrm{GL}(n)$ we put

$$
\begin{aligned}
& g^{-1}=\left(\begin{array}{cccc}
U & W & 0 & 0 \\
V & Z & 0 & 0 \\
0 & 0 & E & F \\
0 & 0 & G & H
\end{array}\right) \begin{array}{l}
\} I_{0} \\
\} I_{0}^{c} \\
I_{1} \\
I_{1}^{c}
\end{array} \\
& \begin{array}{llll}
\tilde{I}_{0} & \tilde{I}_{0}^{c} & \tilde{I}_{1} & \tilde{I}_{1}^{c}
\end{array}
\end{aligned}
$$

Then

$$
p_{I} g^{-1}=\left(\begin{array}{cccc}
U+x_{I} V & W+x_{I} Z & \xi_{I} G & \xi_{I} H \\
\varsigma_{I} V & \varsigma_{I} Z & E+y_{I} G & F+y_{I} H
\end{array}\right)
$$

$\mathscr{V}_{I}$ and $\mathscr{V}_{\tilde{I}}$ are now determined by the conditions $\operatorname{det}\left(U+x_{I} V\right) \neq 0$ and $\operatorname{det}\left(E+y_{I} G\right) \neq 0$, so that

$$
\psi_{I \tilde{I}}=\left(\begin{array}{cc}
U+x_{I} V & \xi_{I} G \\
\varsigma_{I} V & E+y_{I} G
\end{array}\right)
$$

is invertible. Now, writing $\left(\psi_{I \tilde{I}}\right)^{-1}$ in the form

$$
\begin{aligned}
& \left.\left(\psi_{I \tilde{I}}\right)^{-1}=\left(\begin{array}{ll}
\chi_{\tilde{I}_{0} I_{0}} & \chi_{\tilde{I}_{0} I_{1}} \\
\chi_{\tilde{I}_{1} I_{0}} & \chi_{\tilde{I}_{1} I_{1}}
\end{array}\right)\right\} \begin{array}{l}
\tilde{I}_{0} \\
\tilde{I}_{1}
\end{array} \\
& I_{0} \quad I_{1}
\end{aligned}
$$

and using Gauss's decomposition on $\psi_{I \tilde{I}}$ (cf. [Leřtes] and $\S 5$ below), one explicitly finds

$$
\begin{aligned}
& \chi_{\tilde{I}_{0} I_{0}}=\left[\mathrm{id}+\left(U+x_{I} V\right)^{-1} \xi_{I} G\left(E+y_{I} G\right)^{-1} S_{I} V\right]^{-1}\left(U+x_{I} V\right)^{-1}, \\
& \chi_{\tilde{I}_{0} I_{1}}=-\left(U+x_{I} V\right)^{-1} \xi_{I} G\left[\mathrm{id}+\left(E+y_{I} G\right)^{-1} \varsigma_{I} V\left(U+x_{I} V\right)^{-1} \xi_{I} G\right]^{-1} \\
& \times\left(E+y_{I} G\right)^{-1}, \\
& \chi_{\tilde{I}_{1} I_{0}}=-\left(E+y_{I} G\right)^{-1} \zeta_{I} V\left[\mathrm{id}+\left(U+x_{I} V\right)^{-1} \xi_{I} G\left(E+y_{I} G\right)^{-1} \zeta_{I} V\right]^{-1} \\
& \times\left(U+x_{I} V\right)^{-1} \\
& \chi_{\tilde{I}_{1} I_{1}}=\left[\mathrm{id}+\left(E+y_{I} G\right)^{-1} \zeta_{I} V\left(U+x_{I} V\right)^{-1} \xi_{I} G\right]^{-1}\left(E+y_{I} G\right)^{-1} \text {; }
\end{aligned}
$$


and therefore $\rho(g)$ is given in local coordinates by

$$
\begin{aligned}
x_{I} \mapsto & {\left[\mathrm{id}+\left(U+x_{I} V\right)^{-1} \xi_{I} G\left(E+y_{I} G\right)^{-1} \zeta_{I} V\right]^{-1}\left(U+x_{I} V\right)^{-1}\left(W+x_{I} Z\right) } \\
& +\left(U+x_{I} V\right)^{-1} \xi_{I} G\left[\mathrm{id}+\left(E+y_{I} G\right)^{-1} \zeta_{I} V\left(U+x_{I} V\right)^{-1} \xi_{I} G\right]^{-1} \\
& \times\left(E+y_{I} G\right)^{-1} \zeta_{I} Z, \\
\zeta_{I} \mapsto & -\left(E+y_{I} G\right)^{-1} \zeta_{I} V\left[\mathrm{id}+\left(U+x_{I} V\right)^{-1} \xi_{I} G\left(E+y_{I} G\right)^{-1} \zeta_{I} V\right]^{-1} \\
& \times\left(U+x_{I} V\right)^{-1}\left(W+x_{I} Z\right) \\
& +\left[\mathrm{id}+\left(E+y_{I} G\right)^{-1} \zeta_{I} V\left(U+x_{I} V\right)^{-1} \xi_{I} G\right]^{-1}\left(E+y_{I} G\right)^{-1} \zeta_{I} Z, \\
\xi_{I} \mapsto & {\left[\mathrm{id}+\left(U+x_{I} V\right)^{-1} \xi_{I} G\left(E+y_{I} G\right)^{-1} \zeta_{I} V\right]^{-1}\left(U+x_{I} V\right)^{-1} \xi_{I} H } \\
& -\left(U+x_{I} V\right)^{-1} \xi_{I} G\left[\mathrm{id}+\left(E+y_{I} G\right)^{-1} \zeta_{I} V\left(U+x_{I} V\right)^{-1} \xi_{I} G\right]^{-1} \\
& \times\left(E+z_{I} G\right)^{-1}\left(F+y_{I} H\right), \\
y_{I} \mapsto & {\left[\mathrm{id}+\left(E+y_{I} G\right)^{-1} \zeta_{I} V\left(U+x_{I} V\right)^{-1} \xi_{I} G\right]^{-1}\left(E+y_{I} G\right)^{-1}\left(F+y_{I} H\right) } \\
& +\left(E+y_{I} G\right)^{-1} \zeta_{I} V\left[\mathrm{id}+\left(U+x_{I} V\right)^{-1} \xi_{I} G\left(E+y_{I} G\right)^{-1} \zeta_{I} V\right]^{-1} \\
& \times\left(U+x_{I} V\right)^{-1} \xi_{I} H,
\end{aligned}
$$

from which we deduce that $\operatorname{Gr} \rho(g)$ is given by

$$
\begin{aligned}
x_{I} \mapsto & \left(U+x_{I} V\right)^{-1}\left(W+x_{I} Z\right)=-\left(A x_{I}-B\right)\left(C x_{I}-D\right)^{-1}, \\
\zeta_{I} \mapsto- & -\left(E+y_{I} G\right)^{-1} \zeta_{I}\left[V\left(U+x_{I} V\right)^{-1}\left(W+x_{I} Z\right)-Z\right] \\
& =-\left(E+y_{I} G\right)^{-1} \zeta_{I}\left(C x_{I}-D\right)^{-1}, \\
\xi_{I} \mapsto & -\left(U+x_{I} V\right)^{-1} \xi_{I}\left[G\left(E+y_{I} G\right)^{-1}\left(F+y_{I} H\right)-H\right] \\
& =-\left(U+x_{I} V\right)^{-1} \xi_{I}\left(N y_{I}-P\right)^{-1}, \\
y_{I} \mapsto & \left(E+y_{I} G\right)^{-1}\left(F+y_{I} H\right)=-\left(L y_{I}-M\right)\left(N y_{I}-P\right)^{-1},
\end{aligned}
$$

where

are the inverses of

$$
\left(\begin{array}{ll}
A & B \\
C & D
\end{array}\right) \text { and }\left(\begin{array}{cc}
L & M \\
N & P
\end{array}\right)
$$

$$
\left(\begin{array}{cc}
U & W \\
V & Z
\end{array}\right) \text { and }\left(\begin{array}{cc}
E & F \\
G & H
\end{array}\right)
$$

respectively.

These formulas show explicitly that $\operatorname{Gr} \rho(g)$ gives indeed the usual action on the base $G_{k}\left(V_{0}^{m}\right) \times G_{h}\left(V_{1}^{n}\right)$ and on the odd coordinates when regarded as sections of the corresponding tautological bundles. (We shall come back to the group action in $§ 5$.

3. Tautological supervector bundles. It is well known from classical differential geometry that the ordinary grassmannian manifold of $k$-planes in $V_{0}^{m}$, $G_{k}\left(V_{0}^{m}\right)$, can be coordinatized by means of the matrices (cf. (1.9) and assume for the moment that $I_{1}=\varnothing$; hence, $I=I_{0}$ )

$$
p_{I}=\left(\delta_{i a} \mid x_{I}^{i a}\right), \quad i \in I,
$$

with $a \in I$ in the first column and $a \in I^{c}$ in the second. The geometrical interpretation goes as follows: $p_{I}$ is the matrix (with respect to some fixed basis $\left\{\mathbf{e}_{\alpha}\right\}$ of $V_{0}^{m}$ ) of the projection map

$$
p_{I}: V_{0}^{m} \rightarrow W_{I}
$$


onto the $(m-k)$-dimensional subspace $W_{I}:=\operatorname{Span}\left\{\mathbf{e}_{i}: i \in I\right\}$ (see the note in $\S 1$ ). The coordinates $\left(x_{I}^{i a}\right)$ appearing in (3.1) correspond to the $k$-dimensional subspace $\operatorname{Ker} p_{I}$. A basis for this subspace can be taken as

$$
\sigma_{a}^{I}=\sum_{i \in I} x_{I}^{i a} \mathbf{e}_{i}-\mathbf{e}_{a}, \quad a \in I^{c},
$$

and it is understood that $\operatorname{Ker} p_{I}$ belongs to the coordinate chart

$$
\mathscr{U}_{I} \simeq \operatorname{Hom}\left(V_{0}^{m} / W_{I}, W_{I}\right) .
$$

It is then clear that the rank- $k$ tautological vector bundle $E_{k}$ over the grassmannian $G_{k}\left(V_{0}^{m}\right)$ corresponds to the locally free sheaf of $\mathscr{C}^{\infty} \mid G_{k}\left(V_{0}^{m}\right)$-modules defined by letting $\Gamma\left(\mathscr{U}_{I}, E_{k}\right)$ be the $\mathscr{C} \infty\left(\mathscr{U}_{I}\right)$-module freely generated by the $k$ linearly independent sections (3.3).

The transition functions of this bundle are easy to obtain: if $\operatorname{Ker} p_{I}=u \in$ $\mathscr{U}_{I} \cap \mathscr{U}_{J}$, we can use the versions $I$ and $J$ of (3.3) to find a $k \times k$ invertible matrix, $\gamma_{I J}=\left(\gamma_{I J}^{b c}\right)$, such that

$$
\sigma_{c}^{J}(u)=\sum_{b \in I^{c}} \gamma_{I J}^{b c}(u) \sigma_{b}^{I}(u), \quad c \in J^{c},
$$

and in fact,

$$
\left.\gamma_{I J}=\left(\begin{array}{cc}
-x_{J}^{b c} & -x_{J}^{b c} \\
0 & \delta_{b c}
\end{array}\right)\right\} b \in I^{c} \cap J
$$

with $c \in J^{c} \cap I$ in the first column and $c \in J^{c} \cap I^{c}$ in the second, and

$$
\left.\gamma_{I J}^{-1}=\left(\begin{array}{cc}
-x_{I}^{b c} & -x_{I}^{b c} \\
0 & \delta_{b c}
\end{array}\right)\right\} b \in J^{c} \cap I
$$

with $c \in J^{c} \cap I$ in the first column and $c \in J^{c} \cap I^{c}$ in the second. We can similarly describe the other tautological bundle; namely, the rank- $(m-k)$ bundle, $F_{m-k} \rightarrow G_{k}\left(V^{m}\right)$, whose fiber at the point $u \in G_{k}\left(V^{m}\right)$ is given by

$$
F_{u}=\left(V^{m} / u\right)^{*}=\operatorname{Hom}\left(V^{m} / u, \mathbf{R}\right) .
$$

In this case the map $p_{I}: V^{m} \rightarrow W_{I}$ yields an injection $\left(p_{I}\right)^{*}:\left(W_{I}\right)^{*} \rightarrow\left(V^{m}\right)^{*}$ whose image we identify with $F_{u}\left(u=\operatorname{Ker} p_{I}\right)$. Explicitly, let $\left\{\theta^{a}\right\}$ be the dual basis of $\left\{\mathbf{e}_{a}\right\}$ above. Then

$$
\lambda_{I}^{i}:=\left(p_{I}\right)^{*} \theta^{i}=\boldsymbol{\theta}^{i}+\sum_{a \in I^{c}} \boldsymbol{\theta}^{a} x_{I}^{i a}, \quad i \in I,
$$

gives $m-k$ linearly independent sections of $F_{m-k}$ over $\mathscr{U}_{I}$. The transition functions for this bundle are also easy to obtain: this time we let $B(u)=\left(B_{j i}(u)\right)$ be the matrix such that, for $u \in \mathscr{U}_{I} \cap \mathscr{U}_{J}$,

$$
\lambda_{J}^{j}(u)=\sum_{i \in I} \lambda_{I}^{i}(u) B_{j i}(u) .
$$

Again, using (3.8) for $I$ and $J$, one is led to the conclusion that $B$ must satisfy the matrix equation

$$
p_{J}=B \cdot p_{I}
$$


In other words,

$$
B=\left(g_{I J}\right)^{-1}
$$

where $g_{I J}$ is as in (1.12) (under the assumptions $I_{1}=J_{1}=\varnothing$; hence, $I=I_{0}$ and $\left.J=J_{0}\right)$.

Let us now go back to the grassmannian supermanifolds

$$
\mathbf{G}_{k \mid h}\left(V^{m \mid n}\right)=\left(G_{k}\left(V_{0}^{m}\right) \times G_{h}\left(V_{1}^{n}\right), \mathscr{A}\right) .
$$

According to the observations above, it is natural to define the tautological supervector bundle $\mathbf{E}_{k \mid h}$ (resp., $\mathbf{F}_{m-k \mid n-h}$ ) by means of the locally free sheaf of $\mathscr{A}$-modules, $\mathscr{E}_{k \mid h}$ (resp., $\left.\mathscr{F}_{m-k \mid n-h}\right)$, obtained upon the specification that $\mathscr{E}_{k \mid h}\left(\mathscr{U}_{I}\right)$ (resp., $\mathscr{F}_{m-k \mid n-h}\left(\mathscr{U}_{I}\right)$ ) is the free $\mathscr{A}\left(\mathscr{U}_{I}\right)$-bundle generated by the $k$ even sections

$$
\sigma_{a}^{I}=\sum_{i \in I} z_{I}^{i a} \mathbf{e}_{i}-\mathbf{e}_{a}, \quad a \in I_{0}^{c}
$$

and the $h$ odd sections

$$
\tau_{a}^{I}=\sum_{i \in I} z_{I}^{i a} \mathbf{e}_{i}-\mathbf{e}_{a}, \quad a \in I_{1}^{c}
$$

(resp., the $m-k$ even sections

$$
\lambda_{I}^{i}=\boldsymbol{\theta}^{i}+\sum_{a \in I^{c}} \boldsymbol{\theta}^{a} z_{I}^{i a}, \quad i \in I_{0},
$$

and the $n-h$ odd sections

$$
\left.\mu_{I}^{i}=\boldsymbol{\theta}^{i}+\sum_{a \in I^{c}} \boldsymbol{\theta}^{a} z_{I}^{i a}, \quad i \in I_{1}\right)
$$

It is a straightforward matter now to verify that the transition functions for these bundles are given by exactly the same expressions as their classical counterparts, namely,

$$
\begin{aligned}
\gamma_{I J}= & \left.\left(\begin{array}{cc}
-z_{J} & -z_{J} \\
0 & \mathrm{id}
\end{array}\right)\right\} I^{c} \cap J \text { I } \cap I^{c} \text { and } g_{I J}^{-1} \\
& J^{c} \cap I \quad J^{c} \cap I^{c}
\end{aligned}
$$

respectively, where $z_{J}$ has the meaning of (1.10) and $g_{I J}$ that of (1.12).

On the other hand, proceeding as in [OASV 2], one can compute the transition functions of the supercotangent bundle sheaf, $\mathscr{S T}^{*} \mathscr{A}$, of $G_{k \mid h}\left(V^{m \mid n}\right)$. The computation is tedious but straightforward and we obtain, for $j \in J=J_{0} \hat{\cup} J_{1}$ and $i \in I=I_{0} \hat{U} I_{1}$,

$$
\begin{aligned}
& \frac{\partial}{\partial z_{I}^{i a}}\left(g_{J I}^{-1} p_{I}\right)_{j b} \\
& \quad=(-1)^{(|a|+|b|)(|a|+|j|)}\left(g_{I J}^{-1}\right)_{j i} \begin{cases}-z_{J}^{a b}, & a \in I^{c} \cap J, b \in I^{c} \cap J^{c}, \\
-z_{J}^{a b}, & a \in I^{c} \cap J, b \in I \cap J^{c} \\
\delta_{a b}, & a \in I^{c} \cap J^{c}, b \in I^{c} \cap J^{c} \\
0, & a \in I^{c} \cap J^{c}, b \in I \cap J^{c} .\end{cases}
\end{aligned}
$$

In particular, one obtains the following analog of the well-known classical result (for another proof of this fact without using explicitly the transition functions of the supercotangent bundle sheaf, see [Manin]). 
PROPOSITION. There is a natural identification $\mathscr{S T}^{*} \mathscr{A} \simeq \mathscr{F}_{m-k \mid n-h} \otimes \mathscr{E}_{k \mid h}$.

4. Equivariant trivialization theorem. We shall now prove that some supergrassmannians admit the type of global trivialization that makes their structural sheaf look as the sheaf of sections of the exterior algebra bundle of some canonical vector bundle attached to the base manifold. Furthermore, it turns out that for this particular source of examples, such a trivialization is equivariant for the $\mathrm{GL}\left(V_{0}^{m}\right) \times \mathrm{GL}\left(V_{1}^{n}\right)$-action on $\mathrm{G}_{k \mid h}\left(V^{m \mid n}\right.$ ) (given as in $\S 2$ ) and the induced $\mathrm{GL}\left(V_{0}^{m}\right) \times \mathrm{GL}\left(V_{1}^{n}\right)$-action on the space of sections of the bundle in question. Thus, Batchelor's theorem becomes natural for certain examples of supermanifolds (see [Batchelor]), namely, at least those described in the statement of the following

4.1 THEOREM. (a) $\mathbf{G}_{k \mid 0}\left(V^{m \mid n}\right)=\left(G_{k}\left(V_{0}^{m}\right), \mathscr{A}_{G_{k}\left(V_{0}^{m}\right)}\right)$ is a supermanifold of dimension $(k(m-k), n k)$ for which an equivariant identification

$$
\mathscr{A}_{G_{k}\left(V_{0}^{m}\right)} \stackrel{\sim}{\longrightarrow} \mathscr{G}_{r}\left(\mathscr{A}_{G_{k}\left(V_{0}^{m}\right)}\right) \simeq \Gamma\left(\cdot, \bigwedge\left(V_{1} \otimes E_{1}\right)\right)
$$

exists. (Here, $E_{0} \rightarrow G_{k}\left(V_{0}^{m}\right)$ denotes the rank-k tautological vector bundle over the grassmannian $G_{k}\left(V_{0}^{m}\right) ; V_{1}^{n} \rightarrow G\left(V_{0}^{m}\right)$ is the rank-n trivial bundle and $V_{1}^{n} \otimes E_{0} \rightarrow$ $G_{k}\left(V_{0}^{m}\right)$ is the tensor product bundle.)

REMARK. A similar result holds true for the $(k(m-k), n k)$-dimensional supermanifold $\mathbf{G}_{0 \mid k}\left(V^{n \mid m}\right)=\left(G_{k}\left(V_{1}^{m}\right), \mathscr{A}_{G_{k}\left(V_{1}^{m}\right)}\right)$, in which case we have $\mathscr{A}_{G_{k}\left(V_{1}^{m}\right)} \stackrel{\sim}{\longrightarrow}$ $\mathscr{G}_{r}\left(\mathscr{A}_{G_{k}\left(V_{1}^{m}\right)}\right) \simeq \Gamma\left(\cdot, \bigwedge\left(V_{0} \otimes E_{1}\right)\right)$, equivariantly.

(b) $\mathbf{G}_{k \mid n}\left(V^{m \mid n}\right)=\left(G_{k}\left(V_{0}^{m}\right), \mathscr{A}_{G_{k}\left(V_{0}^{m}\right)}\right)$ is a supermanifold of dimension $(k(m-k), n(m-k))$ for which an equivariant identification

$$
\mathscr{A}_{G_{k}\left(V_{0}^{m}\right)} \stackrel{\sim}{\longrightarrow} \mathscr{G}_{r}\left(\mathscr{A}_{G_{k}\left(V_{0}^{m}\right)}\right) \simeq \Gamma\left(\cdot, \bigwedge\left(V_{1} \otimes F_{0}^{*}\right)\right)
$$

exists. (Here, $F_{0}^{*} \rightarrow G_{k}\left(V_{0}^{m}\right)$ denotes the rank- $(m-k)$ tautological vector bundle over the grassmannian $G_{k}\left(V_{0}^{m}\right)$.)

REMARK. Similarly, for $\mathbf{G}_{n \mid k}\left(V^{n \mid m}\right)=\left(G_{k}\left(V_{1}^{m}\right), \mathscr{A}_{G_{k}\left(V_{1}^{m}\right)}\right)$, we have $\mathscr{A}_{G_{k}\left(V_{1}^{m}\right)}$ $\stackrel{\sim}{\longrightarrow} \mathscr{G}_{r}\left(\mathscr{A}_{G_{k}\left(V_{1}^{m}\right)}\right) \simeq \Gamma\left(\cdot, \bigwedge\left(V_{0} \otimes F_{1}^{*}\right)\right)$, equivariantly.

PROOF. We shall prove (a) and (b) simultaneously. The proof itself is divided into two steps; the first consists of convincing ourselves that the sheaves $\mathscr{A}$ and $\mathscr{G} \mathscr{A}$ of the supergrassmannians in question can be identified indeed. To this end, we first use the following lemma.

4.2 LEMMA. For any supermanifold $(M, \mathscr{A})$, we have

$$
\mathscr{G}_{r} \mathscr{A} \simeq \Gamma\left(\cdot, \bigwedge\left(\left(\mathrm{ST}^{*} M\right)_{1}\right)\right),
$$

where $\left(\mathrm{ST}^{*} M\right)_{1}$ is the odd (Whitney) summand of the underlying manifold of the supercotangent bundle (cf. [OASV 2]).

PROOF OF THE LEMMA. This follows essentially from the definition of a supermanifold (cf. [Manin] for details).

We now use the transition functions (3.17) that define the supercotangent bundle sheaf of the supergrassmannians and note that, for $\mathbf{G}_{k \mid 0}\left(V^{m \mid n}\right)\left(\operatorname{resp} ., \mathbf{G}_{k \mid n}\left(V^{m \mid n}\right)\right.$ ), the indexing sequences $I=I_{0} \hat{\cup} I_{1}$ of the covering $\left\{\mathscr{U}_{I}\right\}$ have the following special property:

$$
(\forall I, J) \quad I_{1}=\{1,2, \ldots, n\}=J_{1} \quad \text { and } \quad I_{1}^{c}=\varnothing=J_{1}^{c}
$$




$$
\left[\text { resp., }(\forall I, J) \quad I_{1}=\varnothing=J_{1} \quad \text { and } \quad I_{1}^{c}=\{1,2, \ldots, n\}=J_{1}^{c}\right] .
$$

Therefore, the corresponding matrices $p_{I}$ in (1.11) respectively reduce to

$$
\begin{gathered}
\left.p_{I}=\left(\begin{array}{ccc}
\mathrm{id} & x_{I} & 0 \\
0 & \zeta_{I} & \mathrm{id}
\end{array}\right)\right\} I_{0} \\
I_{1}
\end{gathered}
$$

and

$$
\begin{aligned}
& \left.p_{I}=\left(\begin{array}{lll}
\mathrm{id} & x_{I} & \xi_{I}
\end{array}\right)\right\} I_{0} \\
& \begin{array}{lll}
I_{0} & I_{0}^{c} & I_{1}^{c}
\end{array}
\end{aligned}
$$

Hence, the matrices $g_{I J}$ in (1.12) will take the form

$$
\begin{aligned}
& \left.g_{I J}=\left(\begin{array}{cc}
g_{I_{0} J_{0}} & 0 \\
* & \text { id }
\end{array}\right)\right\} I_{0} \\
& J_{0} \quad J_{1}
\end{aligned}
$$

and

$$
g_{I J}=\left(g_{I_{0} J_{0}}\right)
$$

respectively. In particular,

$$
\begin{gathered}
\left.g_{I J}^{-1}=\left(\begin{array}{cc}
g_{I_{0} J_{0}}^{-1} & 0 \\
* & \mathrm{id}
\end{array}\right)\right\} J_{0} \\
I_{0} \\
I_{1}
\end{gathered}
$$

and

$$
g_{I J}^{-1}=\left(g_{I_{0} J_{0}}^{-1}\right)
$$

Now, we can use these expressions in conjunction with (1.10) and (3.17) to conclude that

$$
\frac{\partial z_{J}^{j b}}{\partial z_{I}^{i a}}=\frac{\partial x_{J}^{i b}}{\partial \zeta_{I}^{i a}} \Leftrightarrow a \in I_{0}, b \in J_{0}, i \in I_{1}, j \in J_{0}
$$

and

$$
\frac{\partial z_{J}^{j b}}{\partial z_{I}^{i a}}=\frac{\partial x_{J}^{j b}}{\partial \xi_{I}^{i a}} \Leftrightarrow a \in I_{0}, b \in J_{0}, i \in I_{0}, j \in J_{0}
$$

respectively. Note right away that (3.17) and (4.4a) immediately imply that

$$
\frac{\partial x_{J}^{j b}}{\partial \varsigma_{I}^{i a}}=0 \quad \text { because }\left(g_{I J}^{-1}\right)_{j i}=0 \quad \text { for all } i \in I_{1}, j \in J_{0} .
$$

On the other hand, for case (b), the only nonvacuous condition on Lemma 4.2 is $a \in I_{1}^{c} \cap J_{1}^{c}$ and $b \in I_{0}^{c} \cap J_{0}^{c}$, in which case $\delta_{a b}=0$. Therefore,

$$
\frac{\partial x_{J}^{j b}}{\partial \xi_{I}^{i a}}=0
$$


Similarly, one notes that

$$
\frac{\partial z_{J}^{j b}}{\partial z_{I}^{i a}}=\frac{\partial \zeta_{J}^{j b}}{\partial \zeta_{I}^{i a}} \Leftrightarrow a \in I_{0}, b \in J_{0}, i \in I_{1}, j \in J_{1}
$$

and

$$
\frac{\partial z_{J}^{j b}}{\partial z_{I}^{i a}}=\frac{\partial \xi_{J}^{j b}}{\partial \xi_{I}^{i a}} \Leftrightarrow a \in I_{1}, b \in J_{1}, i \in I_{0}, j \in J_{0}
$$

For case (a) one sees from (4.4a) that

$$
\left(g_{I J}^{-1}\right)_{j i}=\delta_{i j} \quad \text { for } i \in I_{1}, j \in J_{1} .
$$

Therefore, (3.17) implies that

(4.8a) $\frac{\partial \zeta_{J}^{j b}}{\partial \zeta_{I}^{i a}}=\delta_{i j} \times\left\{\right.$ Transition functions of the rank- $k$ bundle, $\left.E_{0}^{*} \rightarrow G_{k}\left(V_{0}^{m}\right)\right\}$.

On the other hand, for case (b), the only nonvacuous condition in Lemma 4.2 is $a \in I_{1}^{c} \cap J_{1}^{c}$ and $b \in I_{1}^{c} \cap J_{1}^{c}$, in which case,

$$
\frac{\partial \xi_{J}^{j b}}{\partial \xi_{I}^{i a}}=\delta_{a b} \times\left\{\text { Transition functions of the rank- }(m-k) \text { bundle, } F_{0}^{*} \rightarrow G_{k}\left(V_{0}^{m}\right)\right\} \text {. }
$$

But now we are in the situation of having a covering $\left\{\mathscr{U}_{I}\right\}$ for a supermanifold $(M, \mathscr{A})$ with sheaf isomorphisms $\mathscr{A}\left|\mathscr{U}_{I} \rightarrow \mathscr{G}_{r} \mathscr{A}\right| \mathscr{U}_{I}$ satisfying the additional property that the transition functions

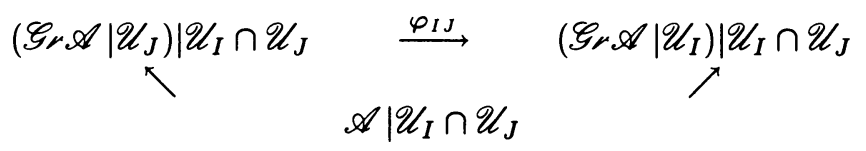

are already of the form $\operatorname{Gr}\left(\varphi_{I J}\right)$ for all $I$ and $J$. (This is precisely the content of equations (4.7) and (4.8) above, as no higher-order terms appear in the transition functions for $x_{J}^{j b}$ and $\xi_{J}^{j b}$ which, in principle, should be polynomials in the odd variables $\xi_{I}^{i b}$.) As the sheaf $\mathscr{A}$ used in the construction of the supergrassmannians was uniquely determined by the transition functions $\varphi_{I J}$, the conclusion is then that we have a global identification $\mathscr{A} \stackrel{\sim}{\longrightarrow} \mathscr{G}_{r} \mathscr{A}$ for both cases (a) and (b). In other words, equations (4.8), together with Lemma 1, imply that

$$
\mathscr{A} \stackrel{\sim}{\longrightarrow} \Gamma\left(\cdot, \bigwedge\left(V_{1}^{n} \otimes E\right)\right)
$$

and

$$
\mathscr{A} \stackrel{\sim}{\longrightarrow} \Gamma\left(\cdot, \bigwedge\left(V_{1}^{n} \otimes F^{*}\right)\right)
$$

hold true globally for the sheaves $\mathscr{A}$ that define the supergrassmannians $\mathbf{G}_{k \mid 0}\left(V^{m \mid n}\right)$ and $\mathbf{G}_{k \mid n}\left(V^{m \mid n}\right)$ respectively.

The second and last part of the proof consists of looking at the action of the group $G=\mathrm{GL}(m) \times \mathrm{GL}(n)$ according to the representation $\rho: G \rightarrow$ Aut $\mathscr{A}$ of $(2.9)$. 
But in view of the properties (4.1) of the indexing sequences $I$ and $J$, the action by $\rho(g)$ with

$$
g=\left(\begin{array}{ccc}
A & B & 0 \\
C & D & 0 \\
0 & 0 & E
\end{array}\right), \quad g^{-1}=\left(\begin{array}{ccc}
U & W & 0 \\
V & Z & 0 \\
0 & 0 & E^{-1}
\end{array}\right)
$$

is reduced in each case to simply

$$
\begin{aligned}
& x_{I} \mapsto\left(U+x_{I} V\right)^{-1}\left(W+x_{I} Z\right)=-\left(A x_{I}-B\right)\left(C x_{I}-D\right)^{-1}, \\
& \varsigma_{I} \mapsto-E_{\zeta_{I}}\left(C x_{I}-D\right)^{-1}
\end{aligned}
$$

and

$$
\begin{aligned}
& x_{I} \mapsto\left(U+x_{I} V\right)^{-1}\left(W+x_{I} Z\right)=-\left(A x_{I}-B\right)\left(C x_{I}-D\right)^{-1}, \\
& \xi_{I} \mapsto\left(U+x_{I} V\right)^{-1} \xi_{I} E^{-1}
\end{aligned}
$$

respectively, which is precisely the action under $\operatorname{Gr}(\rho(g))$.

5. Supergroup action; infinitesimal version. We note that the group action discussed so far is just an ordinary Lie group action. It would be desirable, however, to prove that the supergrassmannians are natural examples of superhomogeneous supermanifolds (in the sense already defined in [Kostant]); that is, that there is a supergroup action defined on them. Theorems regarding superhomogeneous supermanifolds in general shall be postponed for a future work. Here, we shall restrict ourselves to the infinitesimal action for the examples at hand (the supergrassmannians) and get some insight into what to expect in the general case. Along these lines, we should be able to prove the following:

\subsection{PROPOSITION. There is a graded Lie algebra homomorphism}

$$
\tilde{\rho}: \mathfrak{g l}\left(V_{0}^{m} \mid V_{1}^{n}\right) \rightarrow \operatorname{Der} \mathscr{A}_{\mathbf{G}_{k \mid h}\left(V^{m \mid n}\right)}
$$

whose restriction to $\mathfrak{g l}\left(V_{0}^{m}\right) \oplus \mathfrak{g l}\left(V_{1}^{n}\right)=\left(\mathfrak{g l}\left(V_{0}^{m} \mid V_{1}^{n}\right)\right)_{0}$ coincides with the (classical) infinitesimal action obtained from $\S 2$.

The idea of the proof and the actual realization of the action $\tilde{\rho}$ as supervector fields (i.e., superderivations of the structural sheaf $\mathscr{A}$ ) on the supergrassmannian $\mathbf{G}_{k \mid h}\left(V^{m \mid n}\right)$ are based entirely on classical-like arguments. The reason why such classical arguments work on the superhomogeneous setting follows, on the one hand, from Kostant's results [Kostant, $\S \S 3.9,3.10$ ] and, on the other, from our work in [OASV 3] which allows classical interpretations while computing with matrices (see, in particular, $\S 5.3$ below). We shall indicate how an explicit representation of $\mathfrak{g l}\left(V_{0}^{m} \mid V_{1}^{n}\right)$ may be obtained within a coordinate chart and shall give some formulas for the supergrassmannians $\mathbf{G}_{q \mid 0}\left(V^{2 q \mid n}\right)$. A first step in this direction is given by the following rather trivial

5.2 OBSERVATION. Let $\rho: \mathfrak{g l}\left(V_{0} \oplus V_{1}\right) \rightarrow \operatorname{End}\left(M_{0} \oplus M_{1}\right)$ be a representation of the ordinary Lie algebra $\mathfrak{g l}\left(V_{0} \oplus V_{1}\right) \simeq \mathfrak{g l}(m+n)$ into a supervector space $M=M_{0} \oplus M_{1}$. Suppose that

$$
\rho\left(\left(\mathfrak{g l}\left(V_{0} \oplus V_{1}\right)\right)_{0}\right) \subset\left(\operatorname{End}\left(M_{0} \oplus M_{1}\right)\right)_{0}
$$

and

$$
\rho\left(\left(\mathfrak{g l}\left(V_{0} \oplus V_{1}\right)\right)_{1}\right) \subset\left(\operatorname{End}\left(M_{0} \oplus M_{1}\right)\right)_{1}
$$


where

$$
\left(\mathfrak{g l}\left(V_{0} \oplus V_{1}\right)\right)_{0}=\left\{\left(\begin{array}{cc}
A & 0 \\
0 & D
\end{array}\right) \mid A \in \mathfrak{g l}\left(V_{0}\right), D \in \mathfrak{g l}\left(V_{1}\right)\right\}
$$

and

$$
\left(\mathfrak{g l}\left(V_{0} \oplus V_{1}\right)\right)_{1}=\left\{\left(\begin{array}{cc}
0 & B \\
C & 0
\end{array}\right) \mid B \in \operatorname{Hom}\left(V_{1}, V_{0}\right), C \in \operatorname{Hom}\left(V_{0}, V_{1}\right)\right\} .
$$

Then, $\rho$ gives rise to a representation $\tilde{\rho}: \mathfrak{g l}\left(V_{0} \mid V_{1}\right) \rightarrow \operatorname{End}\left(M_{0} \oplus M_{1}\right)$ of the graded Lie algebra $\mathfrak{g l}\left(V_{0} \mid V_{1}\right)$ into the same supervector space $M=M_{0} \oplus M_{1}$.

PROOF OF 5.2. All that is involved in this assertion is the one-to-one correspondence between the representations $\rho$ of $\mathfrak{g l}\left(V_{0} \oplus V_{1}\right)$ and the representations $\rho$ of its universal enveloping algebra $\mathfrak{U}\left(\mathfrak{g l}\left(V_{0} \oplus V_{1}\right)\right)$. In fact, taking into account the vector space isomorphisms

$$
\begin{gathered}
\left(\mathfrak{g l}\left(V_{0} \oplus V_{1}\right)\right)_{0} \simeq \mathfrak{g l}\left(V_{0}\right) \oplus \mathfrak{g l}\left(V_{1}\right) \simeq\left(\mathfrak{g l}\left(V_{0} \mid V_{1}\right)\right)_{0} \\
\left(\mathfrak{g l}\left(V_{0} \oplus V_{1}\right)\right)_{1} \simeq \operatorname{Hom}\left(V_{1}, V_{0}\right) \oplus \operatorname{Hom}\left(V_{0}, V_{1}\right) \simeq\left(\mathfrak{g l}\left(V_{0} \mid V_{1}\right)\right)_{1}
\end{gathered}
$$

and the fact that under the ordinary matrix multiplication (i.e., composition of linear maps)

$$
\left(\mathfrak{g l}\left(V_{0} \oplus V_{1}\right)\right)_{\mu} \cdot\left(\mathfrak{g l}\left(V_{0} \oplus V_{1}\right)\right)_{\nu} \subset\left(\mathfrak{g l}\left(V_{0} \oplus V_{1}\right)\right)_{(\mu+\nu) \bmod (2)},
$$

we can define a degree-zero linear mapping $\tilde{\rho}: \mathfrak{g l}\left(V_{0} \mid V_{1}\right) \rightarrow \operatorname{End}\left(M_{0} \oplus M_{1}\right)$ by letting

$$
\left(\forall x \in \mathfrak{g l}\left(V_{0} \mid V_{1}\right), \text { homogeneous }\right) \quad \tilde{\rho}(x):=\rho(x) \quad(=\rho(x))
$$

and extending it linearly. We now claim that for homogeneous $x, y \in \mathfrak{g l}\left(V_{0} \mid V_{1}\right)$ we have

$$
\tilde{\rho}\left(x \cdot y-(-1)^{|x||y|} y \cdot x\right)=\tilde{\rho}(x) \tilde{\rho}(y)-(-1)^{|x||y|} \tilde{\rho}(y) \tilde{\rho}(x) .
$$

Obviously, it suffices to check this equation for any two odd elements $x, y \in$ $\mathfrak{g l}\left(V_{0} \mid V_{1}\right)_{1}$ (i.e., $\left.|x|=|y|=1\right)$. But in this case we have

$$
\begin{aligned}
\tilde{\rho}(x \cdot y+y \cdot x) & =\boldsymbol{\rho}(x \cdot y+y \cdot x)=\boldsymbol{\rho}(x \cdot y)+\boldsymbol{\rho}(y \cdot x) \\
& =\boldsymbol{\rho}(y) \boldsymbol{\rho}(x)+\boldsymbol{\rho}([x, y])+\boldsymbol{\rho}(x) \boldsymbol{\rho}(y)+\boldsymbol{\rho}([y, x]) \\
& =\boldsymbol{\rho}(y) \boldsymbol{\rho}(x)+\boldsymbol{\rho}(x) \boldsymbol{\rho}(y)=\tilde{\rho}(x) \tilde{\rho}(y)+\tilde{\rho}(y) \tilde{\rho}(x) .
\end{aligned}
$$

5.3. Now, the classical-like argument by which we may arrive at the desired representation $\tilde{\rho}: \mathfrak{g l}\left(V_{0} \mid V_{1}\right) \rightarrow$ Der $\mathscr{A}$ is very simple: choose a local coordinate chart on the supergrassmannian so that (cf. §1)

$$
p=\left(\begin{array}{cccc}
\text { id } & x & 0 & \xi \\
0 & \varsigma & \text { id } & y
\end{array}\right)
$$

Then we choose the supercoset representative

$$
\sigma_{p}=\left(\begin{array}{cccc}
\mathrm{id} & x & 0 & \xi \\
0 & \mathrm{id} & 0 & 0 \\
0 & \varsigma & \mathrm{id} & y \\
0 & 0 & 0 & \mathrm{id}
\end{array}\right)
$$


In order to see what the action of $\mathfrak{g l}\left(V_{0} \mid V_{1}\right)$ looks like in terms of these local coordinates, we first look at the equation

$$
\begin{aligned}
&\left(\begin{array}{llll}
a & b & \alpha & \beta \\
c & d & \gamma & \delta \\
\eta & \varphi & e & f \\
\rho & \tau & g & h
\end{array}\right)\left(\begin{array}{cccc}
1 & x & 0 & \xi \\
0 & 1 & 0 & 0 \\
0 & \zeta & 1 & y \\
0 & 0 & 0 & 1
\end{array}\right) \\
&=\left(\begin{array}{cccc}
1 & g \cdot x & 0 & g \cdot \xi \\
0 & 1 & 0 & 0 \\
0 & g \cdot \varsigma & 1 & g \cdot y \\
0 & 0 & 0 & 1
\end{array}\right)\left(\begin{array}{cccc}
J_{F} & 0 & A & 0 \\
c & J_{E} & \gamma & \Delta \\
N & 0 & J_{H} & 0 \\
\rho & T & g & J_{G}
\end{array}\right)
\end{aligned}
$$

where

$$
g:=\left(\begin{array}{llll}
a & b & \alpha & \beta \\
c & d & \gamma & \delta \\
\eta & \varphi & e & f \\
\rho & \tau & g & h
\end{array}\right) \in \mathrm{GL}\left(V_{0} \oplus V_{1}\right) \simeq \mathrm{GL}(m+n)
$$

is assumed to be close to the identity and $J_{E}, J_{F}, J_{G}$ and $J_{H}$ are elements of $\mathrm{GL}(k), \mathrm{GL}(m-k), \mathrm{GL}(h)$ and $\mathrm{GL}(n-h)$, respectively. Thus, we find that

$$
\begin{aligned}
& g \cdot x=\left\{[(a x+b)+\alpha \zeta]-[(\alpha y+\beta)+a \xi] J_{G}^{-1} T\right\}\left(I-J_{E}^{-1} \Delta J_{G}^{-1} T\right)^{-1} J_{E}^{-1}, \\
& g \cdot \varsigma=\left\{[(\eta x+\varphi)+e \zeta]-[(e y+f)+\eta \xi] J_{G}^{-1} T\right\}\left(I-J_{E}^{-1} \Delta J_{G}^{-1} T\right)^{-1} J_{E}^{-1}, \\
& g \cdot y=\left\{-[(\eta x+\varphi)+e \zeta] J_{E}^{-1} \Delta+[(e y+f)+\eta \xi]\right\}\left(I-J_{G}^{-1} T J_{E}^{-1} \Delta\right)^{-1} J_{G}^{-1}, \\
& g \cdot \xi=\left\{-[(a x+b)+\alpha \varsigma] J_{E}^{-1} \Delta+[(\alpha y+\beta)+a \xi]\right\}\left(I-J_{G}^{-1} T J_{E}^{-1} \Delta\right)^{-1} J_{G}^{-1},
\end{aligned}
$$

where, furthermore,

$$
\begin{aligned}
J_{E} & =(c x+d)+\gamma \zeta, & & T=(\rho x+\tau)+g \varsigma, \\
\Delta & =(\gamma y+\delta)+c \xi, & & J_{G}=(g y+h)+\rho \xi .
\end{aligned}
$$

Note that these calculations are valid under the assumption that $g \in \operatorname{GL}\left(V_{0} \oplus V_{1}\right)$ stays close to the identity, for then we can make sense of $g \cdot x, g \cdot \zeta, g \cdot y$ and $g \cdot \xi$ as coordinates within the same chart. This condition is certainly fulfilled by any smooth curve $t \mapsto g_{t} \in \mathrm{GL}\left(V_{0} \oplus V_{1}\right)$ passing through the identity at $t=0$. In particular, if we set

$$
\left.(d / d t)\left(g_{t}\right)\right|_{t=0}=: \dot{g}_{0} \in \mathfrak{g l}\left(V_{0} \oplus V_{1}\right)
$$

we can differentiate the above formulas for $g_{t} \cdot x, g_{t} \cdot \zeta, g_{t} \cdot y$ and $g_{t} \cdot \xi$ to obtain an expression in local coordinates for the action of the Lie algebra $\mathfrak{g l}\left(V_{0} \oplus V_{1}\right)$ and hence, for the action of the Lie superalgebra $\mathfrak{g l}\left(V_{0} \mid V_{1}\right)$, provided that the conditions of 5.2 are satisfied.

Let us illustrate this construction on the supergrassmannians $\mathrm{G}_{k \mid 0}\left(V^{m \mid n}\right)$. In this case the coordinates $\{y\}$ and $\{\xi\}$ are nonexistent and the above formulas for $g \cdot x$ and $g \cdot s$ reduce to

$$
\begin{aligned}
& g \cdot x=\left\{(a x+b)(c x+d)^{-1}+\alpha \varsigma(c x+d)^{-1}\right\}\left\{I+\gamma \varsigma(c x+d)^{-1}\right\}^{-1} \\
& g \cdot \varsigma=\left\{(\eta x+\varphi)(c x+d)^{-1}+e \varsigma(c x+d)^{-1}\right\}\left\{I+\gamma \zeta(c x+d)^{-1}\right\}^{-1}
\end{aligned}
$$

whenever

$$
g:=\left(\begin{array}{lll}
a & b & \alpha \\
c & d & \gamma \\
\eta & \varphi & e
\end{array}\right) \in \mathrm{GL}\left(V_{0} \oplus V_{1}\right) \simeq \mathrm{GL}(m+n)
$$


Therefore, the corresponding action of $\mathfrak{g l}\left(V_{0} \oplus V_{1}\right)$ will be given by

$$
\begin{aligned}
& X \cdot x=\dot{a} x-x \dot{d}+\dot{b}-x \dot{c} x+\dot{\alpha} \varsigma-x \dot{\gamma} \varsigma \\
& X \cdot \varsigma=\dot{e} \varsigma-\varsigma \dot{d}+\dot{\varphi}-\varsigma \dot{\gamma} \varsigma+\dot{\eta} \varsigma-\varsigma \dot{c} x
\end{aligned}
$$

whenever

$$
X:=\left(\begin{array}{ccc}
\dot{a} & \dot{b} & \dot{\alpha} \\
\dot{c} & \dot{d} & \dot{\gamma} \\
\dot{\eta} & \dot{\varphi} & \dot{e}
\end{array}\right) \in \mathfrak{g l}\left(V_{0} \oplus V_{1}\right) \simeq \mathfrak{g l}(m+n) .
$$

One can now read off from these equations the corresponding action in terms of derivations (with respect to the local coordinates $\left\{x^{i j}\right\}$ and $\left\{\varsigma^{\mu j}\right\}$ ).

To write down some explicit formulas, let us further restrict ourselves to the case $m=2 q$ and $k=m / 2$, so that the latin indices $i, j, \ldots$ will run through $\{1,2, \ldots, q\}$ while the greek indices $\mu, \nu, \ldots$ will run through $\{1,2, \ldots, n\}$. Thus,

$$
\begin{aligned}
& \left(\begin{array}{ccc}
\varepsilon_{i j} & 0 & 0 \\
0 & 0 & 0 \\
0 & 0 & 0
\end{array}\right) \mapsto \sum_{k} x^{j k} \partial_{x^{i k}} \\
& \left(\begin{array}{ccc}
0 & 0 & 0 \\
0 & \varepsilon_{i j} & 0 \\
0 & 0 & 0
\end{array}\right) \mapsto-\sum_{k} x^{k i} \partial_{x^{k j}}-\sum_{\mu} \varsigma^{\mu i} \partial_{\varsigma^{\mu j}} \\
& \left(\begin{array}{ccc}
0 & 0 & 0 \\
\varepsilon_{i j} & 0 & 0 \\
0 & 0 & 0
\end{array}\right) \mapsto-\sum_{k, m} x^{m i} x^{j k} \partial_{x^{m k}}-\sum_{k, \mu} \varsigma^{\mu i} x^{j k} \partial_{\varsigma^{\mu k}}, \\
& \left(\begin{array}{ccc}
0 & \varepsilon_{i j} & 0 \\
0 & 0 & 0 \\
0 & 0 & 0
\end{array}\right) \mapsto \partial_{x^{i j}} \\
& \left(\begin{array}{ccc}
0 & 0 & 0 \\
0 & 0 & 0 \\
0 & 0 & \varepsilon_{\nu \mu}
\end{array}\right) \mapsto \sum_{k} \varsigma^{\mu k} \partial_{\varsigma^{\nu k}} \\
& \left(\begin{array}{ccc}
0 & 0 & \varepsilon_{i \mu} \\
0 & 0 & 0 \\
0 & 0 & 0
\end{array}\right) \mapsto \sum_{k} \varsigma^{\mu k} \partial_{x^{i k}} \\
& \left(\begin{array}{ccc}
0 & 0 & 0 \\
0 & 0 & \varepsilon_{i \mu} \\
0 & 0 & 0
\end{array}\right) \mapsto-\sum_{k, m} x^{m i} \varsigma^{\mu k} \partial_{x^{m k}}-\sum_{k, \nu} \varsigma^{\nu i} \varsigma^{\mu k} \partial_{\varsigma^{\nu k}}, \\
& \left(\begin{array}{ccc}
0 & 0 & 0 \\
0 & 0 & 0 \\
\varepsilon_{\mu i} & 0 & 0
\end{array}\right) \mapsto \sum_{k} x^{i k} \partial_{\varsigma^{\mu k}}, \\
& \left(\begin{array}{ccc}
0 & 0 & 0 \\
0 & 0 & 0 \\
0 & \varepsilon_{\mu i} & 0
\end{array}\right) \mapsto \partial_{\varsigma^{\mu i}} .
\end{aligned}
$$

Note that even (resp., odd) elements of $\mathfrak{g l}\left(V_{0} \oplus V_{1}\right)$ are mapped into even (resp., odd) derivations of the sheaf of local coordinates. Therefore, our observation 5.2 applies and the same correspondences provide us with an explicit realization of the 
superalgebra $\mathfrak{g l}\left(V_{0} \mid V_{1}\right)$ as superderivations of the structural sheaf of the supergrassmannian $\mathbf{G}_{q \mid 0}\left(V^{2 q \mid n}\right)$.

\section{REFERENCES}

[Batchelor] M. Batchelor, The structure of supermanifolds, Trans. Amer. Math. Soc. 253 (1979), 329.

[Boyer and Gitler] C. P. Boyer and Samuel Gitler, The theory of $G^{\infty}$ supermanifolds, Trans. Amer. Math. Soc. 285 (1984), 241-267.

[Corwin, Ne'eman and Sternberg] L. Corwin, Y. Ne'eman and S. Sternberg, Graded Lie algebras in mathematics and physics (Bose-Fermi symmetry), Rev. Mod. Phys. 47 (1975), 573604.

[Guillemin and Sternberg] V. Guillemin and S. Sternberg, Lecture notes on conformal geometry, courses delivered by the authors at Harvard and M.I.T., 1985-1986.

[Jadczyk and Pilch] A. Z. Jadczyk and K. A. Pilch, Superspaces and supersymmetry, Comm. Math. Phys. 78 (1980), 373.

[Kostant] B. Kostant, Graded manifolds, graded Lie theory and prequantization, Lecture Notes in Math., vol. 570, Springer-Verlag, 1977, p. 177.

[LeYtes] D. A. Leĭtes, Introduction to the theory of supermanifolds, Russian Math. Surveys 35 (1980), $1-64$.

[Manin] Yu. Manin, Holomorphic supergeometry and Yang-Mills superfields (preprint).

[Rogers] A. Rogers, A global theory of supermanifolds, J. Math. Phys. 21 (1980), 1352.

[Rothstein 1] Mitchell J. Rothstein, Deformations of complex supermanifolds, Proc. Amer. Math. Soc. 95 (1985), 255.

[Rothstein 2] _ The axioms of supermanifolds and a new structure arising from them, Trans. Amer. Math. Soc. 297 (1986), 159.

[Rothstein 3] _, private communication.

[OASV 1] O. A. Sánchez Valenzuela, Matrix computations in linear superalgebra, Linear Algebra Appl. (to appear).

[OASV 2] _ On supervector bundles (preprint).

[OASV 3] _ Linear supergroup actions. I: On the defining properties, Trans. Amer. Math. Soc. 307 (1988), 569-595.

Instituto de Investigaciones en Matemáticas Aplicadas y Sistemas, Universidad nacional Autónoma de México, Delegacion Alvaro Obregón, 01000 MÉXICO D.F.

Current address: Centro de Investigación en Matematicas, Apartado Postal 402, CP 36000, Guanajuato, Gto, Mexico 